\title{
Comportement mécanique d'un sol grossier à matrice sous cisaillement monotone
}

\section{L.S. PEDRO, J.C. DUPLA} J. CANOU

CERMES (ENPC-LCPC)

6 et 8 , avenue Blaise-Pascal Cité Descartes,

Champs-sur-Marne 77455 Marne-la-Vallée Cedex 2

\section{DORMIEUX}

LMSGC (ENPC-LCPC)

6 et 8 , avenue Blaise-Pascal

Cité Descartes,

Champs-sur-Marne

77455 Marne-la-Vallée

Cedex 2

Y. KAZAN

Université libanaise, Faculté de Génie (LMRS),

Liban
On présente dans cet article les résultats d'une recherché portant sur le comportement mécanique des sols grossiers. Ces matériaux sont caractérisés par une distribution granulométrique très étalée. L'évaluation des caractéristiques mécaniques des sols grossiers est très délicate en raison, en particulier, de la présence des éléments de grande taille. Dans le cadre de cette étude, on se limite au cas des sols grossiers à matrice, il s'agit des sols constitués par de gros éléments entourés par une matrice fine. Le matériau étudié est un sol de référence constitué d'un sable de Fontainebleau auquel sont mélangés des graviers. Après une présentation succincte des dispositifs expérimentaux utilisés dans cette étude, on propose une méthode de fabrication répétable des éprouvettes. Puis on présente des résultats concernant I'influence de la proportion de graviers sur le comportement et sur le critère de rupture du sol hétérogène modèle soumis à un chargement triaxial.

Mots-clés : sol grossier, cisaillement, triaxial, rupture.

We present in this paper the results of a research concerning the mechanical behaviour of coarse grained soils. These materials are characterized by a spread grain size distribution curve. The presence of over sized particles makes difficult the mechanical characterization of these soils. We were more particularly interested in coarse-grained soils composed by rigid inclusions embedded in a fine-grained matrix (heterogenous soil). The studied material is a reference heterogenous soil composed of Fontainebleau sand and gravel. First, we propose a repeatable method of sample fabrication. Then we present some results on the influence of the gravel fraction on the behaviour and on the failure critrion of the soil under a triaxial stress path.

Key words : coarse grained soils, shear, triaxial, failure. 


\section{Introduction}

Les sols grossiers sont des matériaux naturels qui contiennent des éléments de taille très hétérogène. Ils peuvent contenir en effet, des particules de quelques dizaines de microns auxquelles sont mélangés des éléments de plusieurs dizaines de décimètres, voire quelques mètres. On s'intéresse au cas particulier des sols grossiers ayant une granulométrie discontinue, constitués de gros éléments entourés par une matrice d'éléments fins (sable, limon ou argile) : il s'agit des sols grossiers à matrice.

La caractérisation mécanique de ce type de sols peut être réalisée in situ en utilisant des essais dits « en vraie grandeur ») (Shirdam et al., 1998), des dispositifs expérimentaux de grandes dimensions comme les essais à la plaque (Lin et al. ; 2000, Vallé, 2001) et les essais à la boite de cisaillement direct in situ (Laréal et al. 1973; Jain et Gupta, 1974). La caractérisation mécanique en laboratoire nécessite, le plus souvent, l'utilisation de dispositifs de grandes dimensions (Bourdeau, 1997; Shirdam et al., 1998; Vallé, 2001 ; Gomes Correia et al., 2001). En effet, les dispositifs expérimentaux de dimensions plus classiques, requièrent l'écrêtement des éléments de grande taille contenus dans le sol grossier étudié.

L'influence de l'écrêtement sur les caractéristiques mécaniques des sols grossiers constitue donc un point important dans l'étude du comportement mécanique de ces matériaux. En effet, cette reconstitution granulométrique induit une modification de l'hétérogénéité de taille, car les éléments les plus gros sont enlevés. Elle entraîne également une modification de l'hétérogénéité de nature, car l'écrêtement des éléments grossiers s'accompagne naturellement d'une augmentation de la proportion d'éléments fins qui peuvent ètre dans certains cas des limons ou de l'argile. Bien que ce paramètre ait été étudié dans le cadre de travaux de recherche portant sur des matériaux grossiers naturels (Holtz et Gibbs, 1956 ; Donaghe et Thorrey, 1979 ; Gomes Correia et al., 2001 ; Vallé, 2001), les résultats obtenus par les différents auteurs sont souvent contradictoires. Ces différences viennent principalement de la variabilité importante des sols grossiers naturels étudiés (nature, taille...).

Afin d'isoler l'effet de l'hétérogénéité de taille, nous étudions un sol hétérogène modèle constitué uniquement d'éléments granulaires. Ce sol est composé d'un mélange de sable fin ne contenant pas d'éléments limoneux ou argileux, et de graviers.
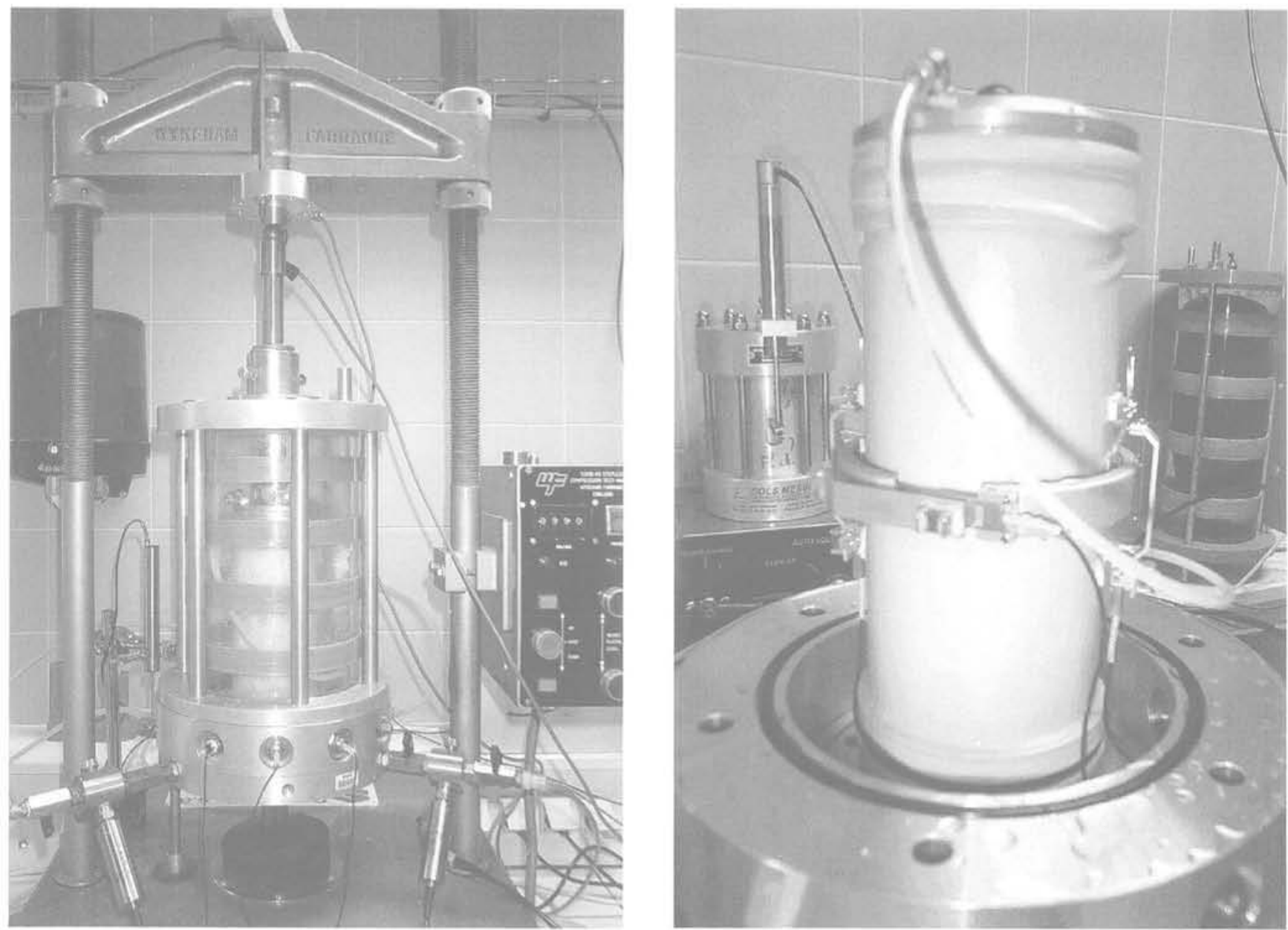

FIG.1 Dispositif expérimental : vue d'ensemble de la cellule triaxiale (a), système de mesure locale des déformations de l'éprouvette (b). 


\section{Procédures expérimentales}

2.1

\section{Dispositifs d'essais}

Le dispositif expérimental (Fig. 1) utilisé pour cette étude est constitué d'une cellule triaxiale permettant de solliciter des éprouvettes cylindriques d'un diamètre de $100 \mathrm{~mm}$ et d'une hauteur de $200 \mathrm{~mm}$. Cette cellule est placée sur une presse ayant une capacité de $50 \mathrm{kN}$. Les essais triaxiaux sont réalisés à taux de déformation contrôlé $(0,5 \%$ par minute) et en condition drainée.

L'éprouvette peut être instrumentée de capteurs de déplacement à effet Hall afin de mesurer localement ses déformations axiales et radiales (Fig. 1b). Dans le cadre de notre étude, la détermination de la déformation axiale de l'échantillon se fait en mesurant le déplacement de l'embase inférieure à l'aide d'un capteur de déplacement longue course $(40 \mathrm{~mm}$, la précision $\pm 0,01 \mathrm{~mm}$ ) (Fig. 1a). L'effort axial est mesuré à l'aicle d'un capteur de force placé à l'intérieur de la cellule $(32 \mathrm{kN}$, précision de $\pm 16 \mathrm{~N})$, son positionnement permet de s'affranchir des effets du frottement entre le piston et le couvercle de la cellule. Un volumètre de marque Wykeham et Farrance d'une capacité de $100 \mathrm{~cm}^{3}$ (précision de $\pm 0,05 \mathrm{~cm}^{3}$ ) permet de mesurer, au cours du cisaillement, les variations de volume de l'éprouvette.

Un logiciel, développé sous Labview, permet de visualiser, au cours de l'essai, les différentes grandeurs mesurées et de les enregistrer dans un fichier pour un traitement ultérieur.

\section{Matériaux constitutifs des éprouvettes testées}

Les éprouvettes sont constituées d'un mélange de deux matériaux naturels. Le premier composant du mélange est un sable de Fontainebleau (SIFRACO) dont les caractéristiques physiques sont répertoriées dans le tableau 1. Ce sable est mélangé en proportion variable à des graviers anguleux (Fig. 2) dont la masse volumique des grains solides est identique à celle du sable $\left(\rho_{5}=2,65 \mathrm{~g} / \mathrm{cm}^{3}\right)$, et dont le diamètre est compris entre 8 et $10 \mathrm{~mm}$. Un rapport de $1 / 10$ entre le diamètre du plus gros élément et celui de l'éprouvette est suffisant pour éviter les effets d'échelle. Le contraste de taille entre les éléments sableux et les graviers est tel que l'on peut clairement dissocier les éléments grossiers des éléments fins. Les graviers peuvent donc être considérés comme des inclusions et le sable comme la matrice.

TABLEAUI Caractéristiques physiques du sable de Fontainebleau.

Physical characteristics of Fontainebleau sand.

\begin{tabular}{c|c|c|c|c}
$\mathrm{D}_{00}(\mathrm{~mm})$ & $\mathrm{C}_{\mathrm{v}}$ & $\mathrm{e}_{\min }$ & $\mathrm{e}_{\min }$ & $\mathrm{r}_{\mathrm{s}}\left(\mathrm{g} / \mathrm{cm}^{3}\right)$ \\
\hline 0,21 & 1,52 & 0,54 & 0,94 & 2,65
\end{tabular}

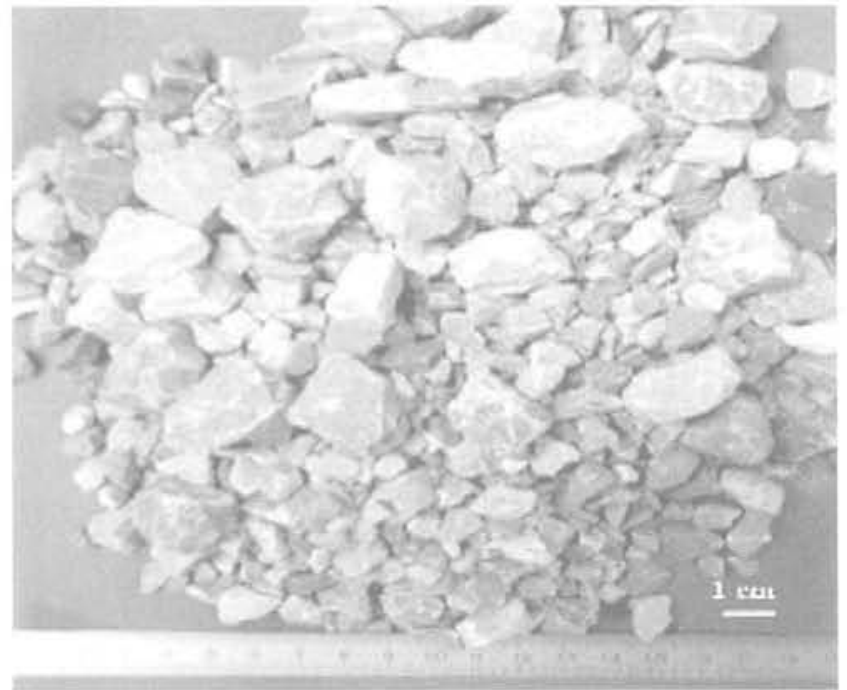

FG. 2. Vue des graviers constituant les éléments grossiers.

View of the gravel material used as inclusion.

\section{3.}

\section{Caractérisation des éprouvettes et description de la procédure de fabrication}

L'état initial de compacité des éprouvettes est défini à partir de la masse volumique moyenne de la matrice sableuse $\rho_{\text {dimat }}$ : quelle que soit la quantité de graviers présente dans l'éprouvette, ce paramètre reste égal à $1,58 \mathrm{~g} / \mathrm{cm}^{3}$. Cette valeur correspond à un état moyennement dense de la matrice (indice de densité de 0,7 ). Le second paramètre qui caractérise une éprouvette de sol hétérogène est la proportion de graviers. On définit à cet effet le paramètre fraction massique de graviers, noté $f_{m}$, de la façon suivante :

$$
f_{\mathrm{m}}=\frac{M_{\mathrm{g}}}{M_{\mathrm{g}}+M_{\mathrm{s}}}
$$

avec : $M_{0}$ : la masse de graviers;

$\mathrm{M}_{5}^{\mathrm{g}}$ : la masse de sable.

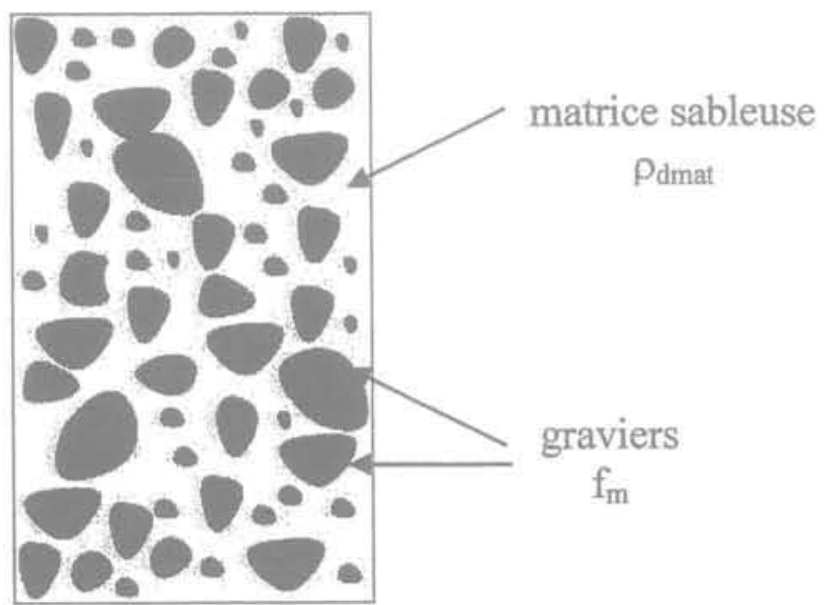

FIG. 3 Caractérisation d'une éprouvette de sol hétérogène.

Characterization of heterogenous soil sample. 
Les éprouvettes sont fabriquées en compactant manuellement 10 couches de sol ayant une hauteur de $2 \mathrm{~cm}$. Chaque couche est constituée d'un mélange de sable et d'une proportion de graviers égale à $\mathrm{f}_{\mathrm{m}}$. Avant d'être introduit dans le moule de préformage de l'éprouvette, le mélange des deux matériaux est humidifié et homogénéisé de façon à prévenir tout phénomène de ségrégation granulaire. On s'assure ainsi d'une répartition (homogène» des graviers au sein de la matrice sableuse,

Afin de pouvoir conclure à un effet significatif des caractéristiques des graviers, il est nécessaire d'évaluer au préalable le degré de répétabilité de fabrication des éprouvettes. Dans ce but, nous avons réalisé quatre essais triaxiaux drainés sur des éprouvettes contenant toutes la même proportion de graviers et le même type de graviers. Ces éprouvettes sont toutes fabriquées selon le procédé décrit ci-dessus. La répétabilité obtenue est satisfaisante (Fig. 4) et permet de valider la procédure expérimentale.

\section{3}

\section{Résultats expérimentaux}

Le programme expérimental consiste à réaliser des essais triaxiaux drainés sur des éprouvettes contenant des proportions variables de graviers. Les valeurs de $f_{m}$ valent respectivement $0,50,75$ et $100 \%$. Pour tous les essais, la matrice se trouve à un indice de densité de 0,7 et la contrainte de consolidation isotrope p' est égale à $100 \mathrm{kPa}$. Le tableau II présente les caractéristiques des essais réalisés dans le cadre de cette étude.

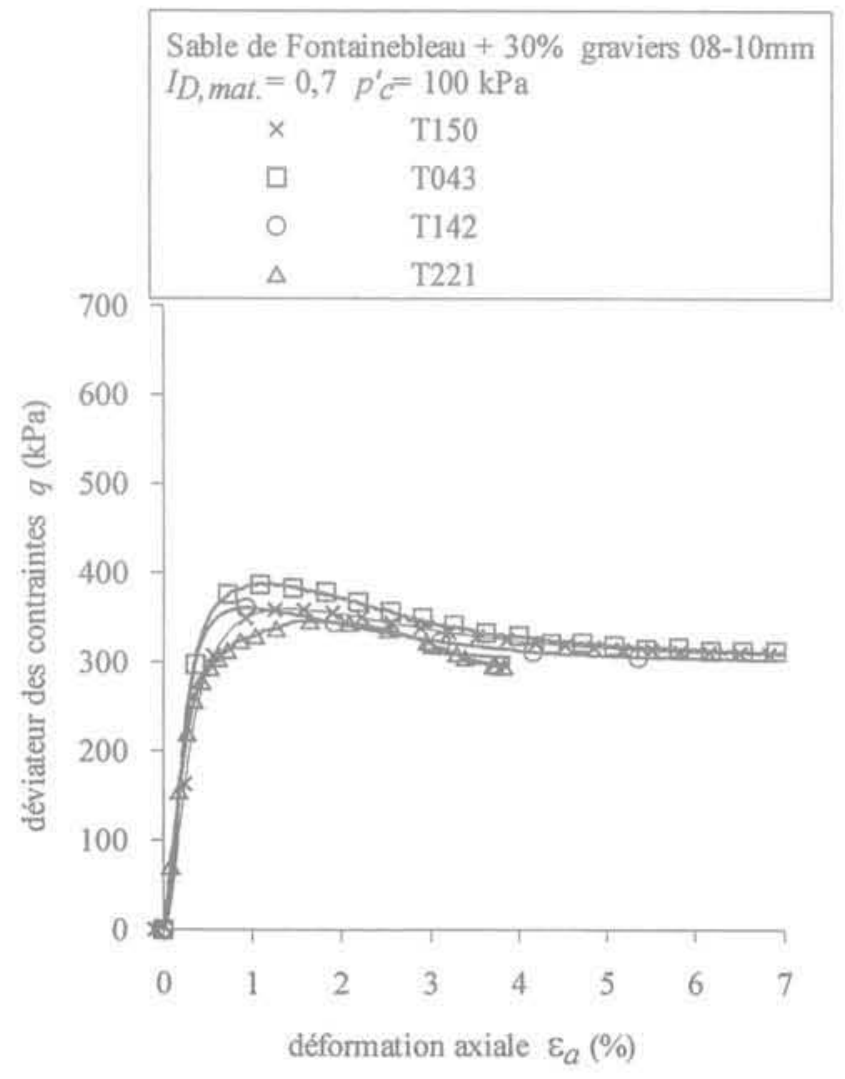

TABLEAUII Caractéristiques des essais triaxiaux drainés réalisés.

Caracteristics of drained triaxial tests realised.

\begin{tabular}{|c|c|c|c|c|c|}
\hline \multirow[t]{2}{*}{ Test } & \multirow[b]{2}{*}{$\begin{array}{c}P_{c}^{\prime} \\
(\mathrm{kPa})\end{array}$} & \multicolumn{2}{|c|}{ Matrice } & \multicolumn{2}{|c|}{ Inclusions } \\
\hline & & Type & $\left(\mathrm{g} / \mathrm{cm}^{3}\right)$ & Type 131 & $\begin{array}{l}f \\
(\%)\end{array}$ \\
\hline T43 & T43 & $\mathrm{Fb}$ & 1,58 & Gr8-10 & 30 \\
\hline T44 & T44 & $\mathrm{Fb}$ & 1,58 & Gr8-10 & 20 \\
\hline T47 & T47 & $\mathrm{Fb}$ & 1,58 & Gr8-10 & 50 \\
\hline T76 & T76 & $\mathrm{Fb}$ & 1,58 & Gr8-10 & 0 \\
\hline T142 & T142 & $\mathrm{Fb}$ & 1,58 & Gr8-10 & 30 \\
\hline T150 & T150 & $\mathrm{Fb}$ & 1,58 & Gr8-10 & 30 \\
\hline T153 & T153 & $\mathrm{Fb}$ & 1,58 & Gr8-10 & 75 \\
\hline T154 & T154 & $\mathrm{Fb}$ & 1,58 & Gr8-10 & 100 \\
\hline T221 & $\mathrm{T} 221$ & $\mathrm{Fb}$ & 1,58 & Gr8-10 & 30 \\
\hline
\end{tabular}

(1) $\mathrm{Fb}$ : sable de Fontainebleau

2) Gr8-10: graviers 8-10 mm.

\section{1}

\section{Mobilisation du cisaillement}

Lorsque la proportion de graviers augmente, on observe (Fig. 5) un accroissement de la valeur du dévia-

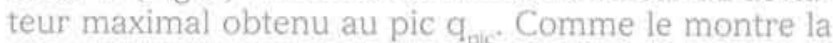
figure 6, l'évolution du paramètre $\mathrm{q}_{\text {pic }}$ est non linéaire et semble se stabiliser pour des valeurs élevées de la proportion de graviers (supérieures à $50 \%$ ). On constate

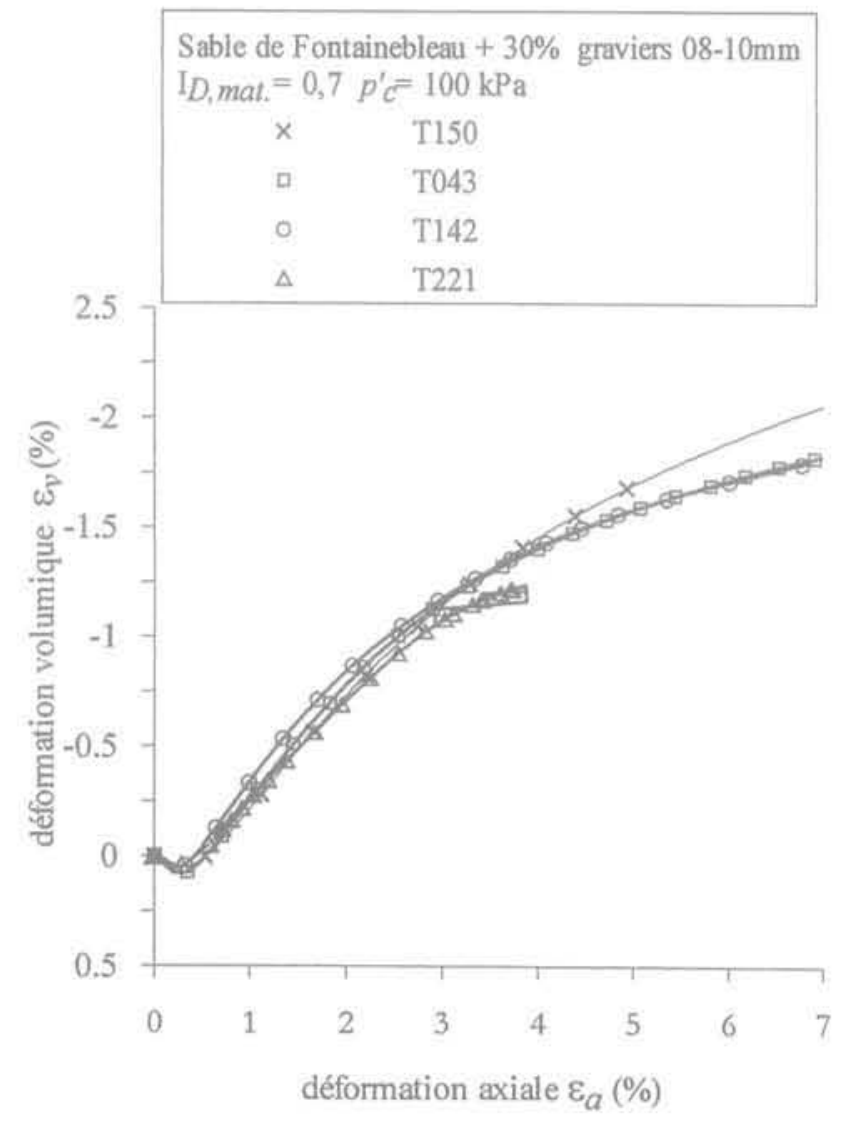

FG. 4 Essais de répétabilité : courbes de cisaillement et courbes de déformation volumique. Repetability tests: shearing curves and volumic strain curves. 


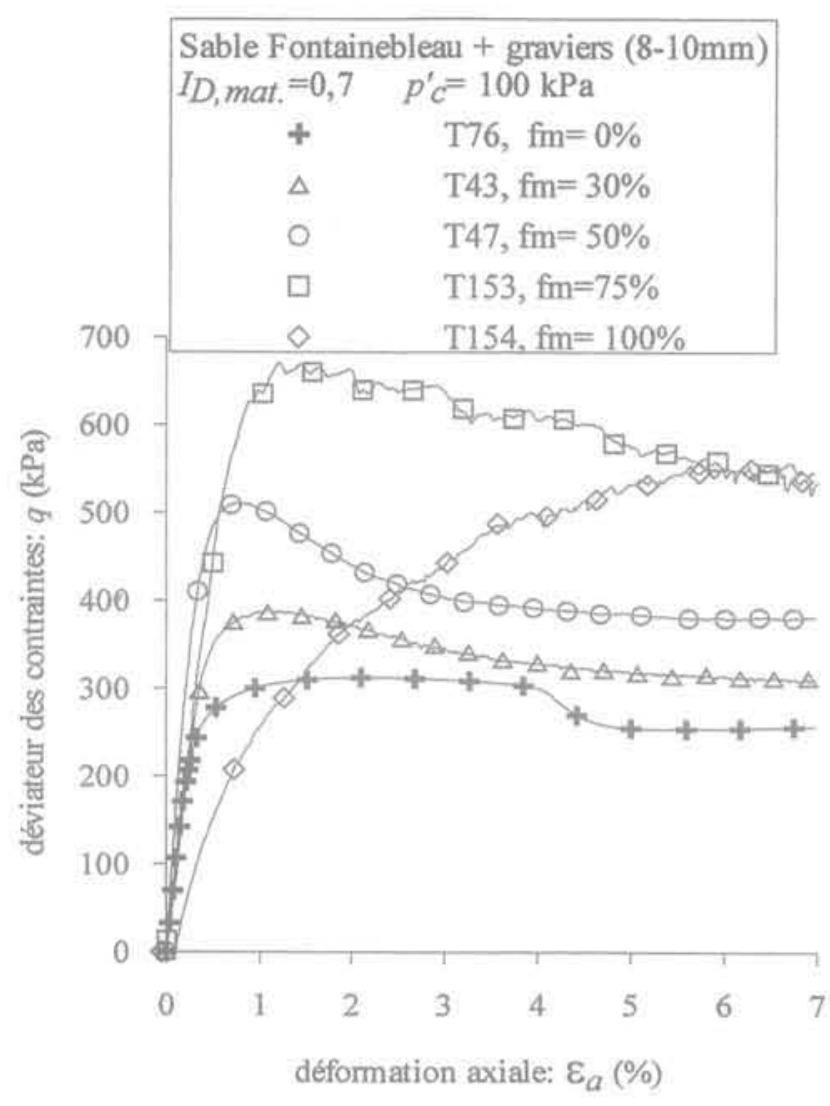

FG. 5 Influence de la fraction massique de graviers sur les courbes de cisaillement.

Influence of gravel fraction on the shearing curves:

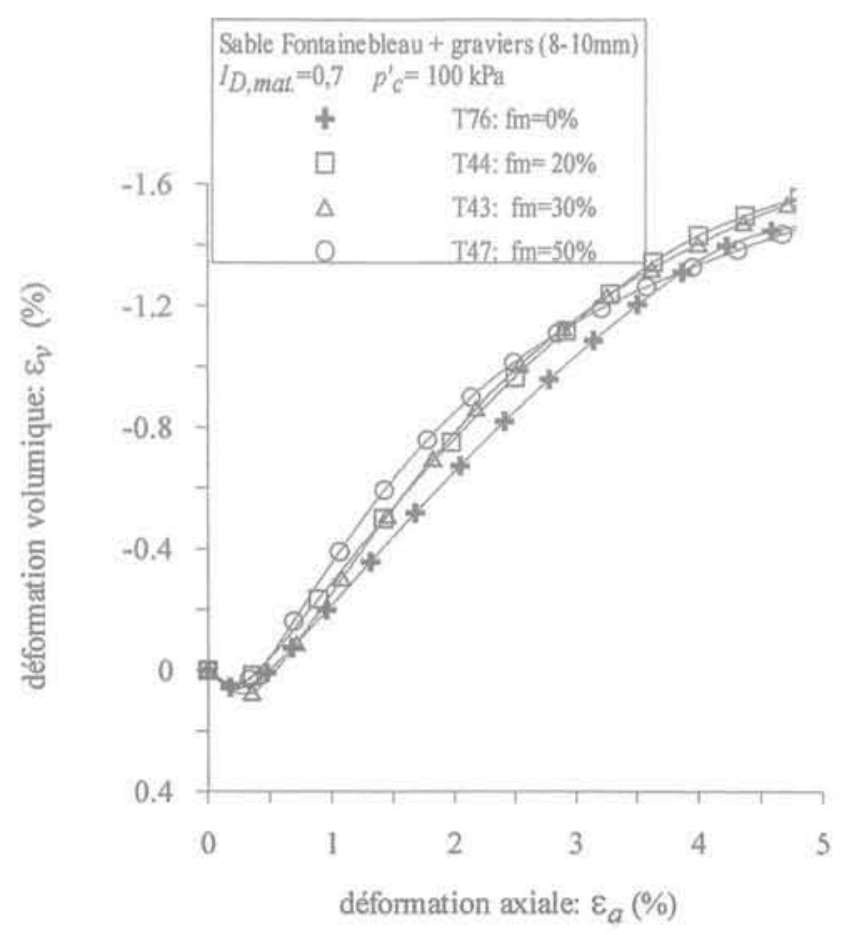

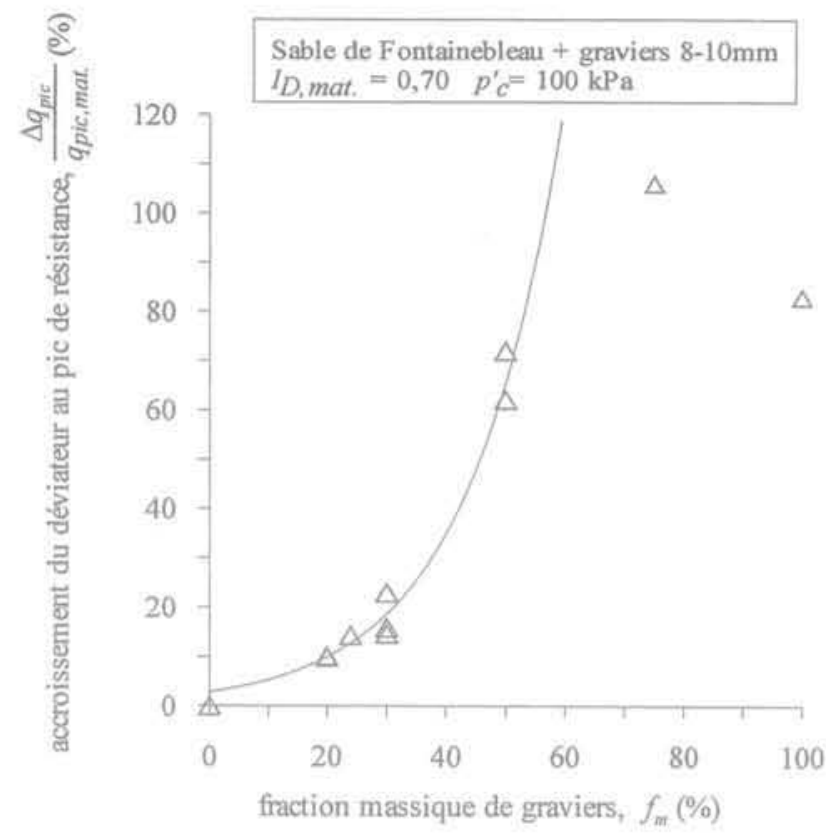

FG. 6 Influence de la fraction massique de graviers sur le déviateur à la rupture.

Influence of gravel fraction on the failure deviatoric stress.

également que l'éprouvette contenant $100 \%$ de graviers présente une raideur initiale nettement plus faible que celle des autres éprouvettes.

L'effet de renfort observé est lié à la présence des graviers, qui constituent, au sein de l'éprouvette, des éléments rigides reprenant les efforts de cisaillement. La transmission de ces efforts est possible car les graviers, ayant une surface rugueuse, adhèrent à la matrice sableuse par frottement aux interfaces matrice-graviers.

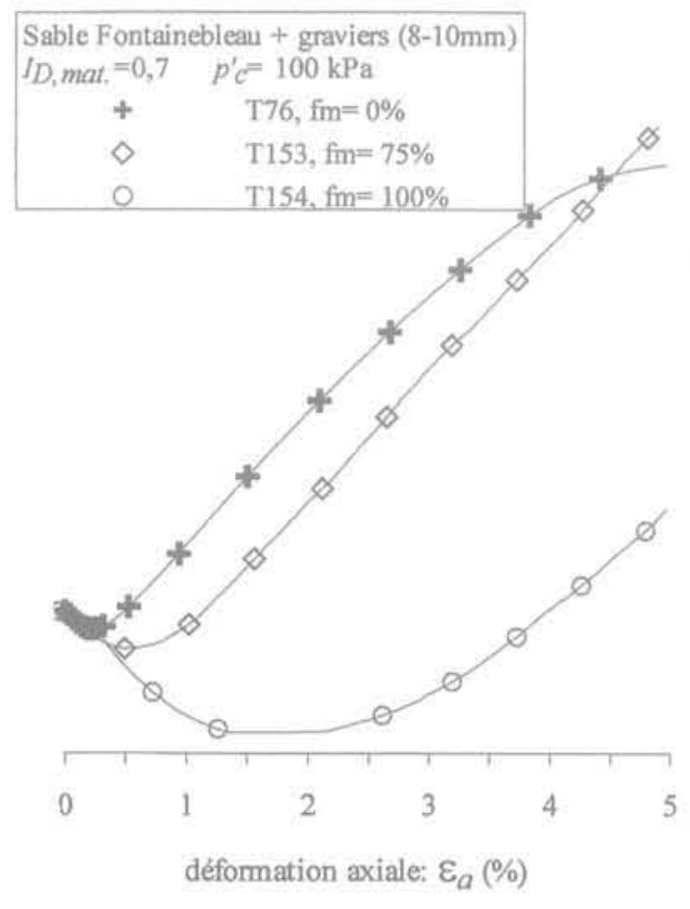

FG. 7 Influence de la fraction massique de graviers sur la déformation volumique de l'éprouvette. Influence of gravel fraction on the sample volumic strain curves. 
Dans le cas des proportions d'inclusions élevées (75\% et $100 \%$ ), l'effet de renfort semble être dû principalement aux forces de contact entre les graviers. La diminution de la valeur de $\mathrm{q}_{\text {pic }}$ pour la fraction massique de $100 \%$ s'explique par le fait que, les vides entre les graviers ne contiennent pas de matériau. La résistance au cisaillement de cette éprouvette est exclusivement liée aux propriétés de contact entre les graviers. L'absence de matrice sableuse entre les graviers explique également la diminution de la raideur de l'éprouvette contenant $100 \%$ de graviers.

\section{Déformations volumiques}

La figure 7 montre que plus la proportion de graviers augmente, plus l'éprouvette semble avoir un comportement dilatant. En effet, on peut observer sur la figure 8 , que le taux de dilatance $\alpha\left(\mathrm{d} \varepsilon_{2} / \mathrm{d} \varepsilon_{0}\right)$ augmente en fonction de $f_{n t}$. Il semble que cette variation du paramètre a ne traduise pas une augmentation du potentiel de dilatance de l'éprouvette. Elle traduit plutôt le fait que lorsque la proportion de graviers croît, la fraction de matière déformable (matrice sableuse) diminue. Cette explication est confirmée par le caractère linéaire de la relation entre $\alpha$ et $f_{m} \%$ (Fig. 8)

Pour des valeurs plus élevées de la proportion de graviers, on note une brusque chute du taux de dilatance. Pour ces valeurs de $\mathrm{f}_{\mathrm{m}}$, comme nous l'avons évoqué plus haut, la structure granulaire est constituée de graviers en contact entre eux et de sable remplissant plus ou moins les vides entre les graviers. Ainsi, le potentiel de dilatance de l'éprouvette ne dépend plus de celui de la matrice sableuse, mais de celui du squelette granulaire constitué par les graviers.

L'allure des courbes de variation de volume nous renseigne sur l'initiation de la localisation des déformations de l'éprouvette. En comparant par exemple les courbes de variation de volume d'une éprouvette ne contenant aucune inclusion à celle d'une éprouvette qui en contient $30 \%$ (Fig. 9), on note l'existence

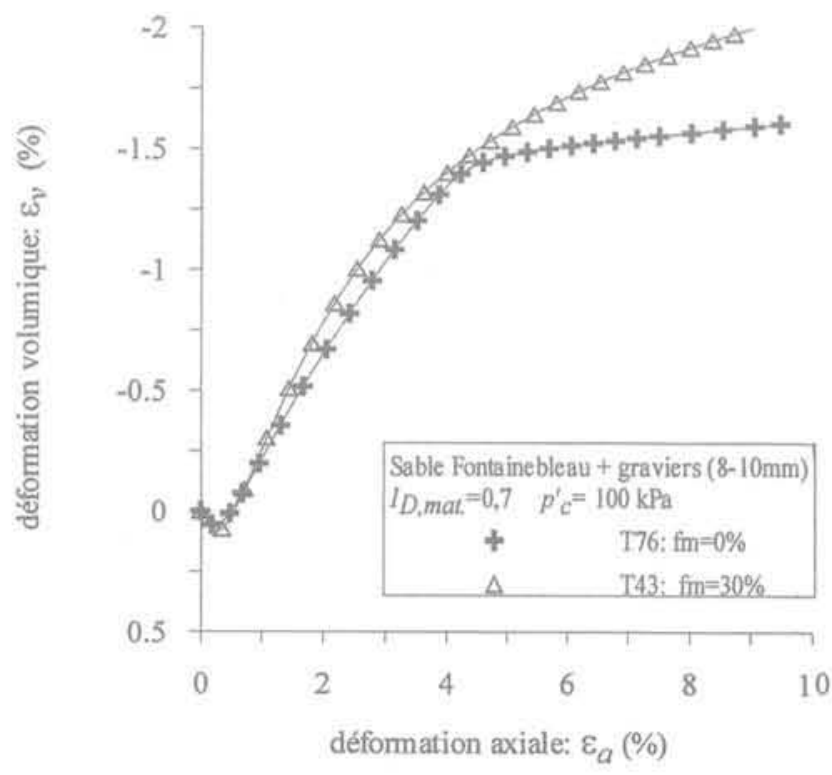

d'un changement brusque d'évolution de la courbe pour l'éprouvette sans inclusions. La présence de ce coude correspond à l'occurrence du phénomène de localisation des dêformations. Par contre, les courbes des essais réalisés sur les éprouvettes contenant 30\% de graviers ne présentent pas de coude : tout se passe comme si la présence des graviers entravait le développement d'une bande de cisaillement. Les photos d'éprouvettes (Fig. 9) après essais corroborent bien cette interprétation : on voit en effet que l'éprouvette sans graviers s'est rompue en deux blocs, alors qu'il n'existe pas de bande de cisaillement nette sur l'éprouvette contenant des graviers.

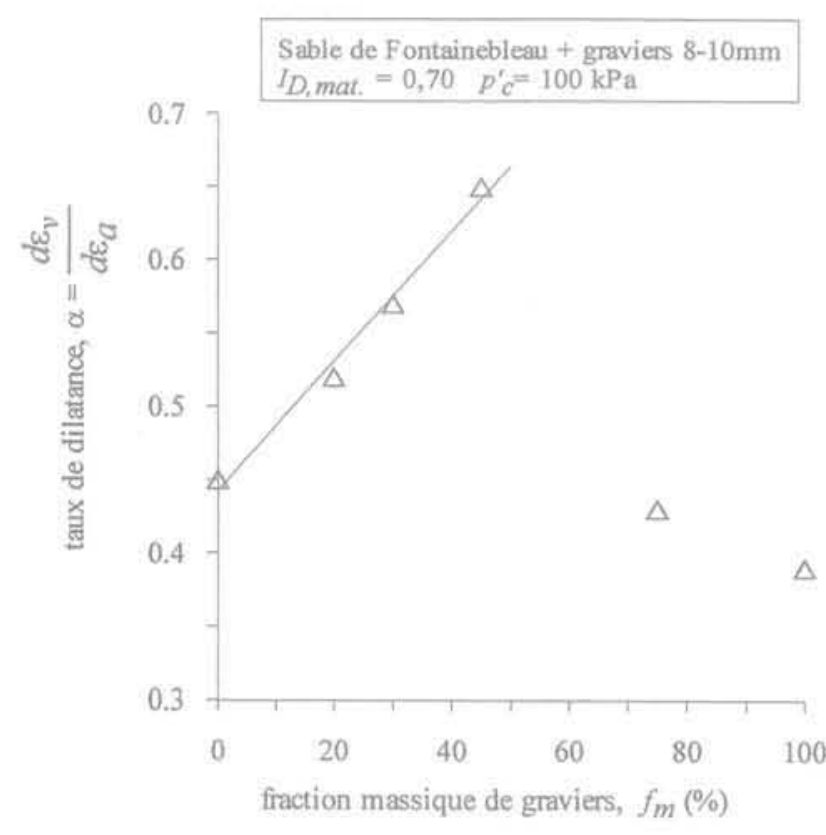

FIG. 8 Influence de la fraction massique de graviers sur le taux de dilatance. influence of gravel fraction on dilatancy ratio.
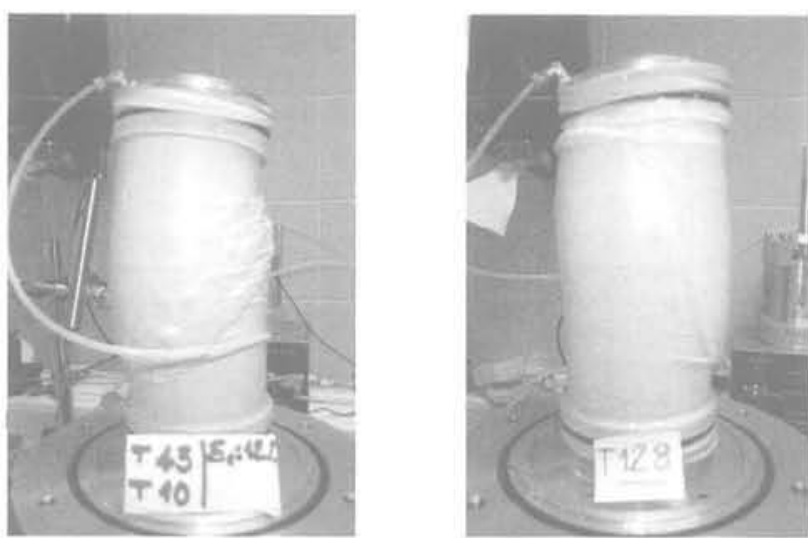

FG. 9 Influence de la présence des graviers sur le mode de rupture. Influence of gravel on failure mode, 


\section{Recherche d'un critère de rupture}

Pour atteindre cet objectif, nous avons réalisé des essais triaxiaux à diverses valeurs de la pression de consolidation isotrope : $\mathrm{p}_{\mathrm{c}}^{\prime}=50,100$ et $200 \mathrm{kPa}$. Pour étudier l'évolution du critère en fonction de la proportion de gravier, un jeu d'essais a été réalisé pour différentes proportions de gravier.

Nous avons représenté dans le plan (q, p') les points expérimentaux correspondants aux différents essais (Fig, 10). On constate d'abord, que l'augmentation de la proportion de gravier n'a pas d'influence sur le caractère linéaire du critère de rupture. Ensuite, on remarque qu'en ajoutant des éléments grossiers dans une matrice pulvérulente, le mélange ainsi obtenu est également dénué de cohésion. Finalement, on note que l'angle de frottement des éprouvettes est une fonction croissante de la proportion de graviers (Fig. 11).

\section{4}

\section{Conclusion}

Les travaux présentés ont permis d'isoler l'influence de l'hétérogénéité de taille sur les caractéristiques de rupture d'un sol grossier modèle. Ils ont montré l'existence d'un effet d'accroissement de la résistance au cisaillement dû à la présence d'éléments rigides au sein d'une matrice plus fine et moins rigide. Cette recherche a également mis en évidence une modification du comportement du sol hétérogène à partir d'une certaine valeur de la proportion d'éléments grossiers. En effet,

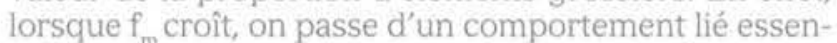
tiellement aux caractéristiques de la matrice, à un comportement complètement gouverné par les graviers. Ce changement de comportement est notable en termes d'effort mobilisé et de variation de volume.

Ces résultats peuvent être utilisés dans le cadre pratique qui est celui de la déduction des caractéristiques mécaniques d'un sol hétérogène à partir de celles du sol écrêté. Ces travaux démontrent en effet que l'écrêtement des éléments grossiers conduit à une sous-estimation de propriétés mécaniques des sols grossiers hétérogènes ne contenant pas de fines (argiles ou limons). Par ailleurs, il semble que les caractéristiques mécaniques du sol hétérogène peuvent être évaluées à partir des propriétés de la matrice et de la proportion d'éléments grossiers. En effet, nous avons mis en évidence de façon expérimentale, des relations qui relient les caractéristiques mécaniques du sol étudié ( $\mathrm{q}_{\mathrm{pje}}$ taux de dilatance et angle de frottement) à celles de ces composants (matrice sableuse et éléments grossiers).

L'étude du comportement mécanique d'un sol grossier modèle apporte des éléments d'éclaircissement sur l'influence de l'hétérogénéité de taille qui est une des principales caractéristiques des sols grossiers. L'influence d'autres paramètres significatifs tels que la taille, la forme des éléments grossiers a fait l'objet de récents travaux. L'effet de l'état initial a été également étudié (Pedro, 2004). Néanmoins, il reste à étendre cette étude au cas des mélanges granulaires ayant une granulométrie continue ainsi qu'au cas de matériaux naturels qui ont une composition plus complexe et plus variée que celle du sol modèle.

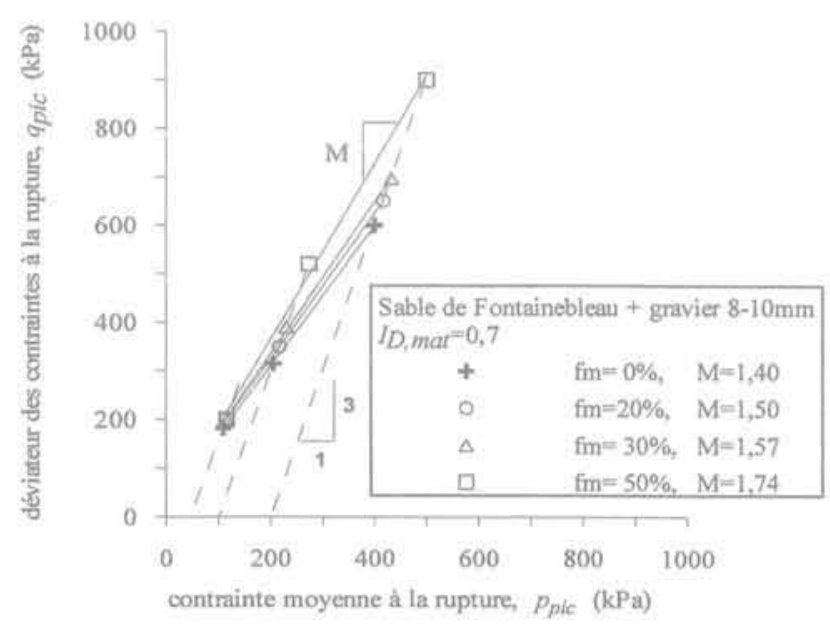

FIG. 10 Influence de la fraction massique de graviers sur la droite de rupture. Influence of gravel fraction on the failure criterion.
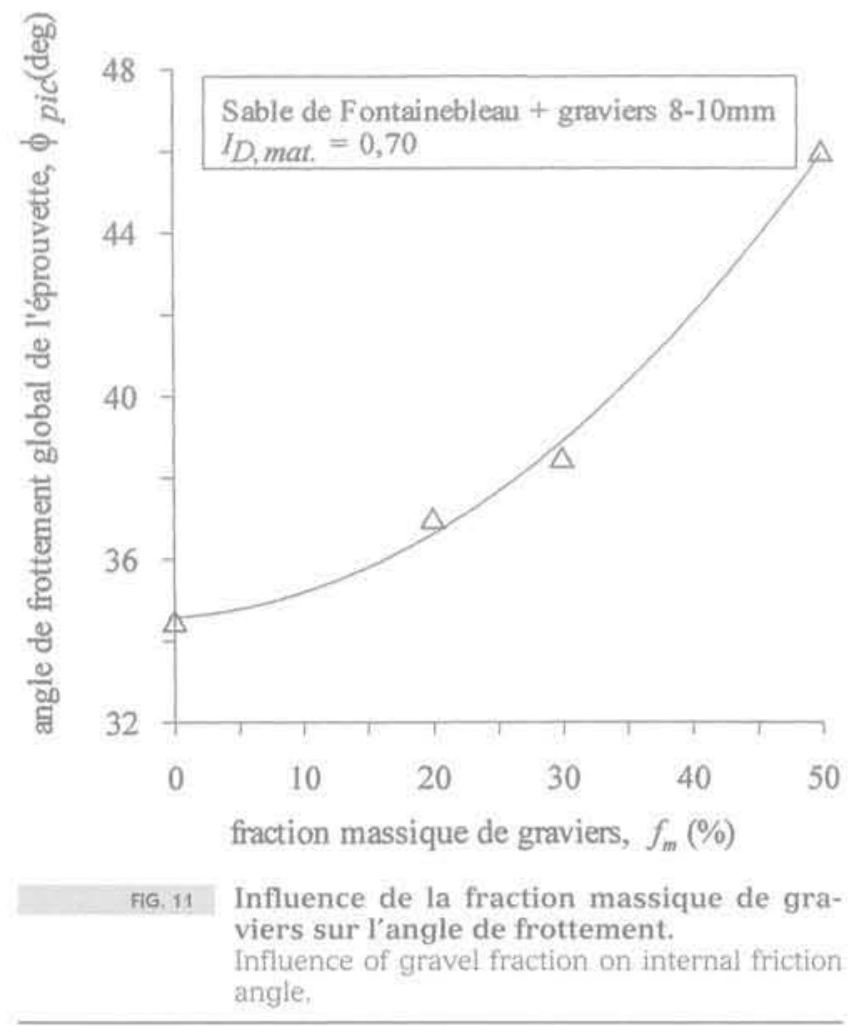
Bourdeau Y. - Le comportement des alluvions du Rhône dans une grande boîte cisaillement direct. Revue française de géotechnique $\mathrm{n}^{\circ} 79,1997, \mathrm{p}$. 45-57.

Donaghe R.T., Torrey V.H. - Scalping and replacement effects on strencths parameters of earth-rock mixtures. Design parameters in geotechnical engineering, London, 1979, vol. 2, p. 29-34

Gomes Correja A., Dan L.K., Koseki J., Tast. suoka F. - Small strain stiffness under different isotropic and anisotropic stress conditions of two granular granite materials. Advanced Laboratorystress-Strain Testing of Geomaterials. 2001, vol. 1, p. 209-215.
Holtz W.G., Gibbs H.J. - Triaxial shear test on previous gravelly soils. Journal of the Soil Mechanics and Foundation of the ASCE, vol. 82. 1956, p. 1-9

Jain S.P, Gupta R.C. - In situ shear test for rockfills. Journal of the Soil Mechanics and Foundation of the ASCE, vol. 100. 1974, p. 1031-1050.

Laréal P., Bourdeau Y., Lambert P. Cottereau $C$. - Essais de cisaillement in situ sur les sols alluvionnaires de la vallée du Rhóne. Travaux, vol. 55, 1973, p. $52-59$

Lin S.Y. Lin P.S., Luo H.S., Juang C.H. Shear modulus and damping ratio characteristics of gravelly deposit.
Canadian Geotechnical Journal, vol. 37, 2000, p. 638-651.

Pedro L.S. - De l'étude du comportement mécanique de sols hétérogènes modèles à son application au cas des sols naturels. Thèse de doctorat de 'ENPC, 2004

Shirdam R., Faure R.M., Magnan J.-P. Caractéristiques des éboulis de pente à l'aide d'une boite de cisaillement. The Geotechnics of Hard Soils-Soft Rocks, Picarelii \&Evangelista (eds), Balkema. Rotterdam. 1998, p. 869-880.

Vallé N. - Propriétés mécaniques d'un sol grossier d'une terrasse alluvionnaire de la Seine. Thèse de doctorat de Yuniversité de Caen, 2001. 